\title{
A novel fiber-optic pressure sensor device for measuring variceal pressure
}

\author{
XI WANG ${ }^{1 *}$, HUIXIAN LI ${ }^{1 *}$, JING WANG $^{1}$, AIJIU WU ${ }^{2}$ and DERUN KONG ${ }^{1}$ \\ ${ }^{1}$ Department of Gastroenterology, Anhui Geriatric Institute, The First Affiliated Hospital of Anhui Medical University; \\ ${ }^{2}$ Anhui University of Architecture, Hefei, Anhui 230022, P.R. China
}

Received December 1, 2018; Accepted September 13, 2019

DOI: $10.3892 /$ etm.2019.8071

\begin{abstract}
It has been reported that variceal pressure can predict the occurrence of variceal bleeding. However, the majority of methods presently used to measure variceal pressure are either invasive or inconvenient. In the present study, a fiber-optic pressure sensor was constructed to detect variceal pressure. The prospective study focused on the in vitro accuracy of a fiber-optic pressure sensor and investigated the clinical reliability and feasibility of this method. The fiber-optic pressure sensor covered a pressure-sensitive probe containing a fiber-optic pressure sensor and a workstation to record the pressure tracing. It was hypothesized that the endoscopic fiber-optic pressure sensor can effectively predict the risk of variceal bleeding. To test this hypothesis, 80 patients who suffered from cirrhosis and who had a history of variceal bleeding were included in the present study. The fiber-optic pressure sensor was guided through the biopsy channel using an endoscope in the patient cohort. Transjugular intrahepatic stent-shunt (TIPS) was subsequently performed within $24 \mathrm{~h}$ after measuring variceal pressure. A comparison of the results of the 80 patients was made between variceal pressure measured by the endoscopic fiber-optic pressure sensor and the portal pressure gradient (PPG) measured by a TIPS. The variceal pressure measurements with the fiber-optic pressure sensor were technically satisfactory in 78 patients. The results indicated that there was a linear correlation between the variceal pressure measured by the endoscopic fiber-optic pressure sensor and the PPG $(r=0.940, P<0.001)$. These observations suggest that the fiber-optic pressure sensor is an accurate and feasible measurement technique. Therefore, the results of the
\end{abstract}

Correspondence to: Dr Derun Kong, Department of Gastroenterology, Anhui Geriatric Institute, The First Affiliated Hospital of Anhui Medical University, 218 Jixi Road, Hefei, Anhui 230022, P.R. China

E-mail: kongderun168@163.com

*Contributed equally

Key words: variceal pressure, portal venous pressure, fiber-optic pressure sensor present study indicate that the endoscopic fiber-optic pressure sensor is effective in predicting the risk of variceal bleeding.

\section{Introduction}

Esophageal variceal bleeding is a life-threatening complication of cirrhotic portal hypertension (1). According to LaPlace's law of fluid mechanics: Tension of the blood vessel wall=(intravascular pressure-extravascular pressure) $\mathrm{x}$ blood vessel diameter/blood vessel thickness. The intravascular pressure is the root factor for esophageal variceal bleeding and is directly associated with portal pressure. Therefore, variceal pressure serves a crucial role in predicting variceal bleeding and evaluating the effect of drug therapy for portal hypertension (2). Regularly measuring varicose pressure is imperative for patients with cirrhosis. Variceal pressure is currently detected by directly puncturing the varix with a fine needle; however, it is necessary to improve on the methods presently available as they are associated with a high risk of bleeding due to the level of invasiveness. Numerous clinical trials have been conducted in order to investigate measurement techniques that are less invasive, or are even non-invasive, however, the risk of bleeding remains a possibility for these measurements (3-8). A previous research group developed a novel device, termed an endoscopic fiber-optic pressure sensor (9). Compared with traditional pressure sensors, this device uses the theory of fluid conduction pressure (Pascal's law) and a computer workstation (i.e. a data processing terminal) to perform the analysis, and the device processes and creates a graphical display of the pressure grating signal. The investigators herein observed that the device was more convenient than conventional methods, as it can be placed over the varix through an endoscopic biopsy channel. It was hypothesized that the endoscopic fiber-optic pressure sensor can effectively predict the risk of variceal bleeding. Therefore, the present prospective study focused on the accuracy of fiber-optic pressure sensor in vitro and investigated the clinical reliability and feasibility of this method by comparing the results of variceal pressure measured using the fiber-optic pressure sensor and the PPG.

\section{Materials and methods}

In order to measure varicose vein pressure with a fiber-optic pressure sensor in the present study, a measuring device was 
designed consisting of a probe, optical fiber and a measurement workstation. The device was based on a fiber-optic pressure sensor, was designed and constructed by Jiuhong Medical Instrument Co. Ltd. (Changzhou, China), and consisted of a pressure-sensitive probe comprised of a fiber-optic pressure sensor (FOP-F125, FISO Technologies, Inc., Quebec, QC, Canada) and workstation to record the pressure tracing. The fiber-optic probe was inserted through the biopsy channel of an endoscope when the endoscope reached the distal end of the esophagus. The operation method is illustrated in Fig. 1.

Basic structure and detection principle of the probe. The probe consisted of a rigid cylindrical, sealed cavity with a wedge-shaped front end. The wedge plane uses a thin membrane to form a detection surface, and the cavity is equipped with a fiber-optic pressure sensor and fluid medium. The external pressure perceived from the detection surface is transmitted to the sensor through the fluid medium, and the pressure variable is then transformed into a grating signal by the sensor and is transmitted to the workstation interface through the optical fiber $(9,10)$.

When the probe detection surface is fitted to the outer wall of the blood vessel in which pressure is being measured, stress deformation of the detection surface occurs, and along with the filling shape of the outer wall of the vessel (the outward convex surface). The detection surface then deforms inwards. When deformation of the fitted interface remains static, the blood pressure in the vessel will be equal to the fluid pressure in the probe, thus the blood pressure is detected. Pressure analysis during measurement is illustrated in Fig. 2.

There is: $\mathrm{FZY}=\operatorname{Sin} \theta \mathrm{FZ}$

Where FZ is the surface tension of the blood vessel when the probe was pressed againt the blood vessel. The direction of FZ is parallel to the axis of the blood vessel. FZY is the component of FZ in the $\mathrm{Y}$ direction and the value of FZY depends on $\theta, \theta$ is the angle between FZ and the surface of probe detection. The measurement process used by the probe is via internal stress transfer. When the probe reaches and presses the outer wall of the blood vessel, the detection membrane of the probe and the outer wall of the blood vessel deform to the interface in a natural state of static balance. According to Newton's law, the force acting on a body at rest is equal to the reaction force: $F^{\prime}=-F+\sum F Z Y$. As FZY is smaller than $F$, then $F^{\prime} \approx-F$. Simultaneously, the correlation area $(S)$ between the probe membrane and the outer wall of the blood vessel is equal. According to $\mathrm{P}=(\mathrm{F} / \mathrm{S})$, it can be deduced that $p^{\prime} \approx p$. Therefore, the fluid medium pressure $\left(p^{\prime}\right)$ in the probe is almost equal to the blood pressure (p). This process can be further explained by quantitative analysis.

According to Pascal's law, $\mathrm{P}=\rho \mathrm{gh}$, and the fluid density formula, $\rho=(\mathrm{m} / \mathrm{v})$, the following relation can be derived: $\mathrm{P}=\rho \mathrm{gh}=(\mathrm{m} / \mathrm{v}) \mathrm{gh}$. Where $\mathrm{p}$, is the particle pressure in the probe; $\mathrm{g}$ is the acceleration of gravity; $\mathrm{h}$ is the depth of the particle in the probe (the height of the particle from the natural plane of the fluid); $\rho$ is the density of the fluid medium; $m$ is the mass of the fluid medium and $\mathrm{v}$ is the volume of the fluid medium (the probe chamber space).

As the probe cavity is small, the influence of gravity acceleration $(\mathrm{g})$ and particle depth $(\mathrm{h})$ on the measurement process is not considered; i.e. gh is considered to be a physical constant.
When the surface of the probe attached to the outer wall of the blood vessel is deformed by the stress at the surface caused by the internal pressure of the blood vessel, this culminates in depression and deformation of the probe surface, which causes the space of the probe cavity to shrink. This small change and the variation of the fluid pressure in the probe can be derived by differentiating the upper form: $(\delta \mathrm{p} / \delta \mathrm{v})=(\mathrm{p})^{\prime}=[(\mathrm{m} / \mathrm{v}) \mathrm{gh}]^{\prime}=$ -mgh $\left(1 / \mathrm{v}^{2}\right)$.

The deformation of the probe surface is only affected by the internal pressure of the blood vessel and is theoretically unaffected by other external forces, such as those applied at the time of fitting. Any marginal change in the cavity space caused by deformation of the probe surface causes a mathematical correlation difference to the particle pressure of the fluid medium. According to the principle of fluid isotropy, the particle pressure perceived by the sensor in the probe is equal to that of any particle pressure in the detection surface.

Detection process. The detection process began with inserting the probe into the endoscope channel and guiding it to the detection site using an endoscope. The second step was to set the output level of the display terminal of the workstation to zero and enter the measurement state. The probe was then moved closer to the outer wall of the blood vessel so that the detection surface and the outer surface of the blood vessel were parallel, and pressure was gradually applied. The pressure curve and value in the display require monitoring; when the value is stable, that is, when the curve fluctuation slows down and the plateau wave peak appears, the value is the real-time pressure value. During the measurement procedure, as long as the probe surface and the surface of the outer wall of the blood vessel are smoothly fitted, the magnitude of the press pressure will not cause abnormal fluctuation of the force curve. All measured data were saved in the workstation. The force curve in the display is presented in Fig. 3.

Clinical investigation. Between December 2013 and December 2015, the device was used to assess patients with cirrhosis who were scheduled to receive a transjugular intrahepatic stent-shunt (TIPS). All patients had cirrhosis with a history of episodes of esophageal variceal bleeding and presented with large esophageal varices (F2, F3) on endoscopy. The exclusion criteria were total portal vein thrombosis, previous endoscopic treatment of varices (sclerotherapy or endoscopic band ligation) to prevent any additional bleeding, isolated gastric or ectopic varices, previous surgical portosystemic shunt or TIPS, hepatocellular carcinoma or other malignancies, severe clotting defects and grade III or IV hepatic encephalopathy.

A total of 93 eligible patients were enrolled in the present study; 13 patients did not participate resulting in a total of 80 patients. A total of 64 patients suffered from hepatitis B or C. In accordance with the Japanese Research Society for Portal Hypertension (11), the degree of varices in the present study were set at F2 $(n=22)$ or F3 $(n=58)$. The patient characteristics are listed in Table I. The diagnosis of cirrhosis was obtained on the grounds of clinical history, laboratory testing, imaging examinations and an endoscopy.

In order to avoid the artifacts caused by esophageal peristalsis, preoperative preparation (diazepam $5 \mathrm{mg}$; butylbromide 


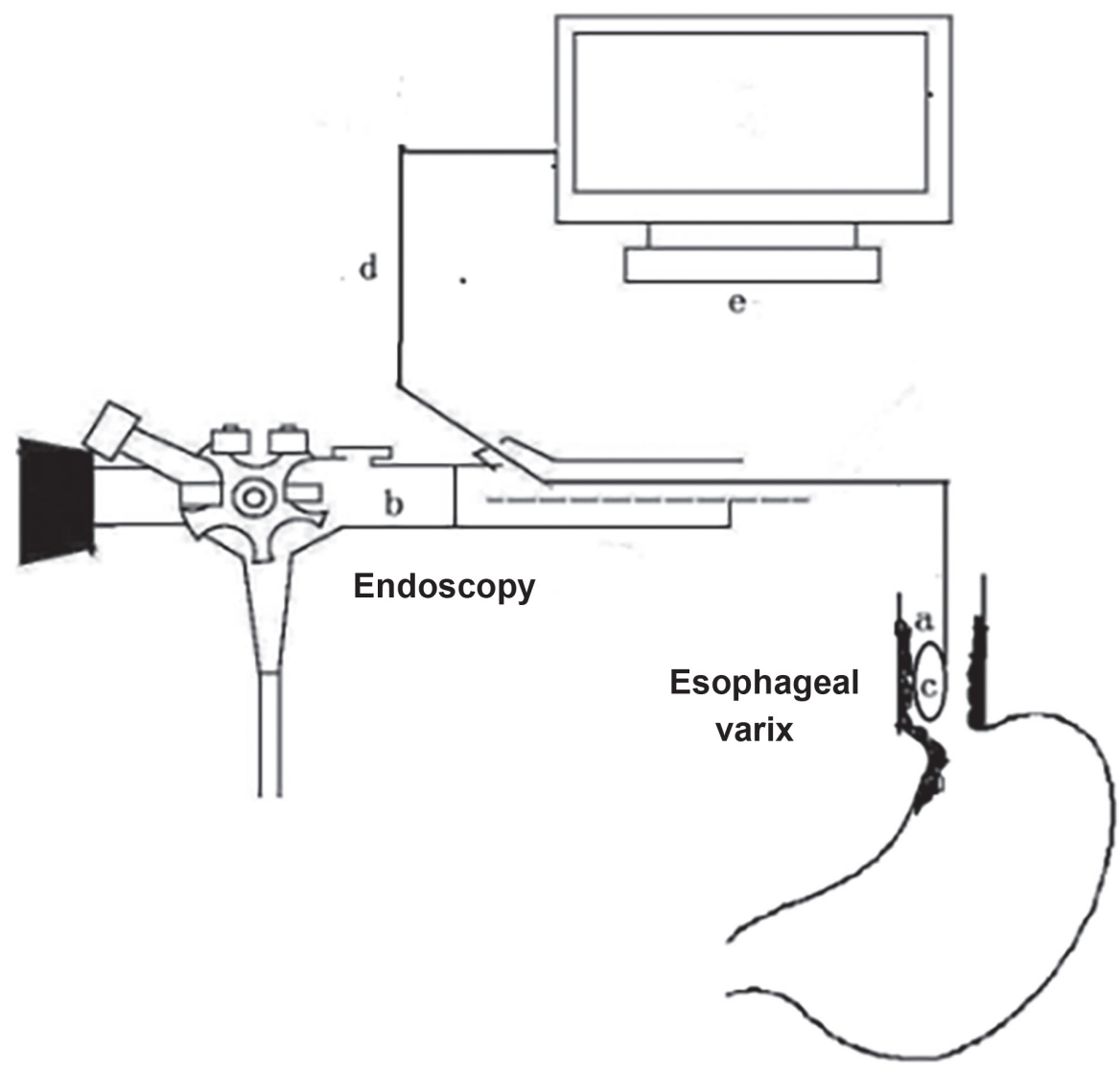

Figure 1. Diagrammatic sketch of the fiber-optic variceal pressure measuring system. (a) Esophageal varix; (b) endoscopy; (c) fiber-optic sensor, inserted through the biopsy channel of the endoscope and placed onto the esophageal varix; (d) optical fiber; (e) workstation, pressure tracings are registered on the computer system.

scopolamine $20 \mathrm{mg}$ ) was required. The aforementioned device was used to measure variceal pressure. The largest varix located above the cardia was detected, once the probe was placed at the distal esophagus through the endoscopic biopsy channel. In order to acquire a stable variceal pressure tracing, the probe was applied over the largest varix for $5 \mathrm{sec}$ during the intermission of two peristaltic waves.

The criteria required for a sustained satisfactory measurement included a zero-pressure point and a stable variceal pressure tracing for at least $5 \mathrm{sec}$, with the probe applied over the varix under direct visual control. A pressure point of zero was defined as the atmospheric pressure in the esophagus and prior to each measurement, the starting pressure was adjusted to zero. In addition to the above criteria, satisfactory variceal pressure measurements were also required to meet the following predetermined criteria: i) Absence of artifacts caused by esophageal contractions and ii) correct placement of the probe over the varix. The mean value of five satisfactory measurements was used to determine the variceal pressure. In order to eliminate observer bias, the variceal pressure was measured by two independent observers. The mean of five satisfactory measurements from each observer was used to determine the mean variceal pressure of every patient and ensure intraand inter-observer agreement.

TIPS was performed within $24 \mathrm{~h}$ after variceal pressure measurement. The technique approach was as described in a previous publication (9). A A7F pigtail catheter was introduced into the inferior vena cava under fluoroscopic guidance. The free hepatic venous pressure (FHVP) was recorded when the tip of the catheter floated at the junction of the hepatic vein and the inferior vena cava. The portal vein pressure (PVP) was measured when the portal venous was punctured under the guiding of digital subtraction angiography using the RUPS 100 set (RUPS-100, Cook Inc., Bloomington, IL, USA). The PPG resulted from the difference between the PVP and FHVP in the occluded TIPS condition. To ensure that PPG measurements were satisfactory, the pressure tracings remained stable for at least $30 \mathrm{sec}$. The mid-chest was used as the external zero reference. Following placement of an 8-mm stent (BARD, Luminexx, Voisins le Bretonneux, France) to generate the TIPS, the PPG of the open TIPS condition was recorded, and the second variceal pressure measurement was recorded following the TIPS procedure.

Statistical analysis. Quantitative data are presented as the mean \pm standard deviation. Significant differences between the control and treated groups were determined using an unpaired Student's t-test. Inter-observer correlation was analyzed using Pearson's correlation coefficient and intra-observer correlation was analyzed using the intraclass correlation coefficient. All analyses were conducted using SPSS 16.0 software (SPSS, Inc., Chicago, IL, USA). P $<0.05$ was considered to indicate a statistically significant difference. 


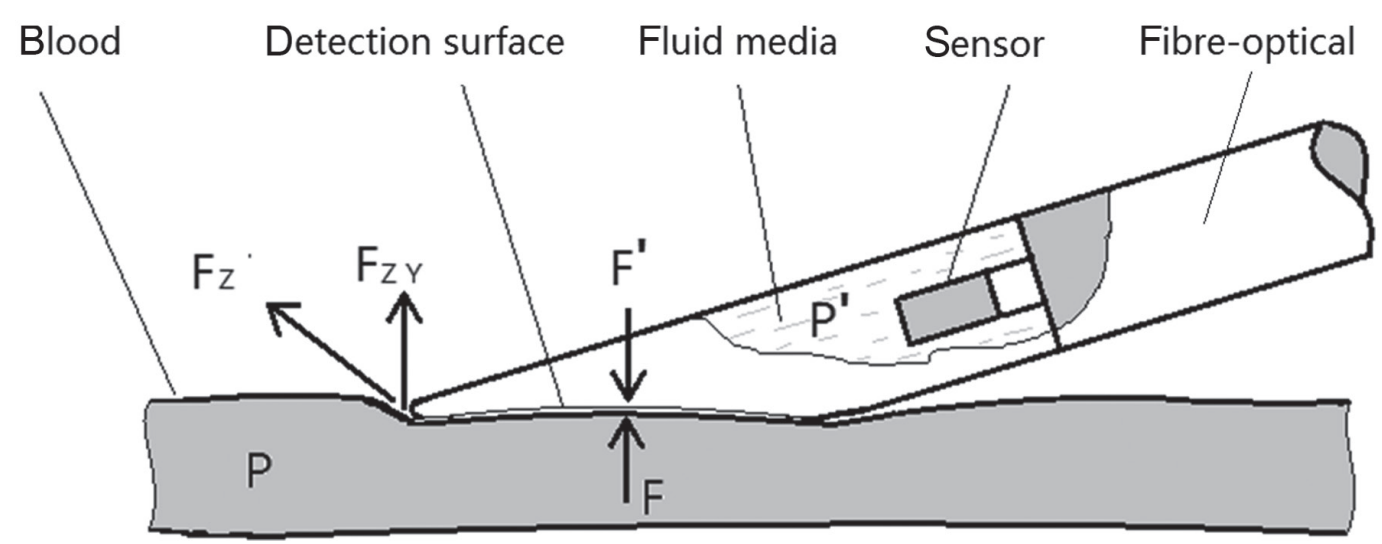

Figure 2. Probe and detection system. F, internal pressure of the blood vessel; F', reverse force of the probe fluid medium; FZ, surface tension of the blood vessel around the outer diameter of the probe; FZY, Y component of the surface tension; P, blood pressure; $\mathrm{P}^{\prime}$, fluid medium pressure.

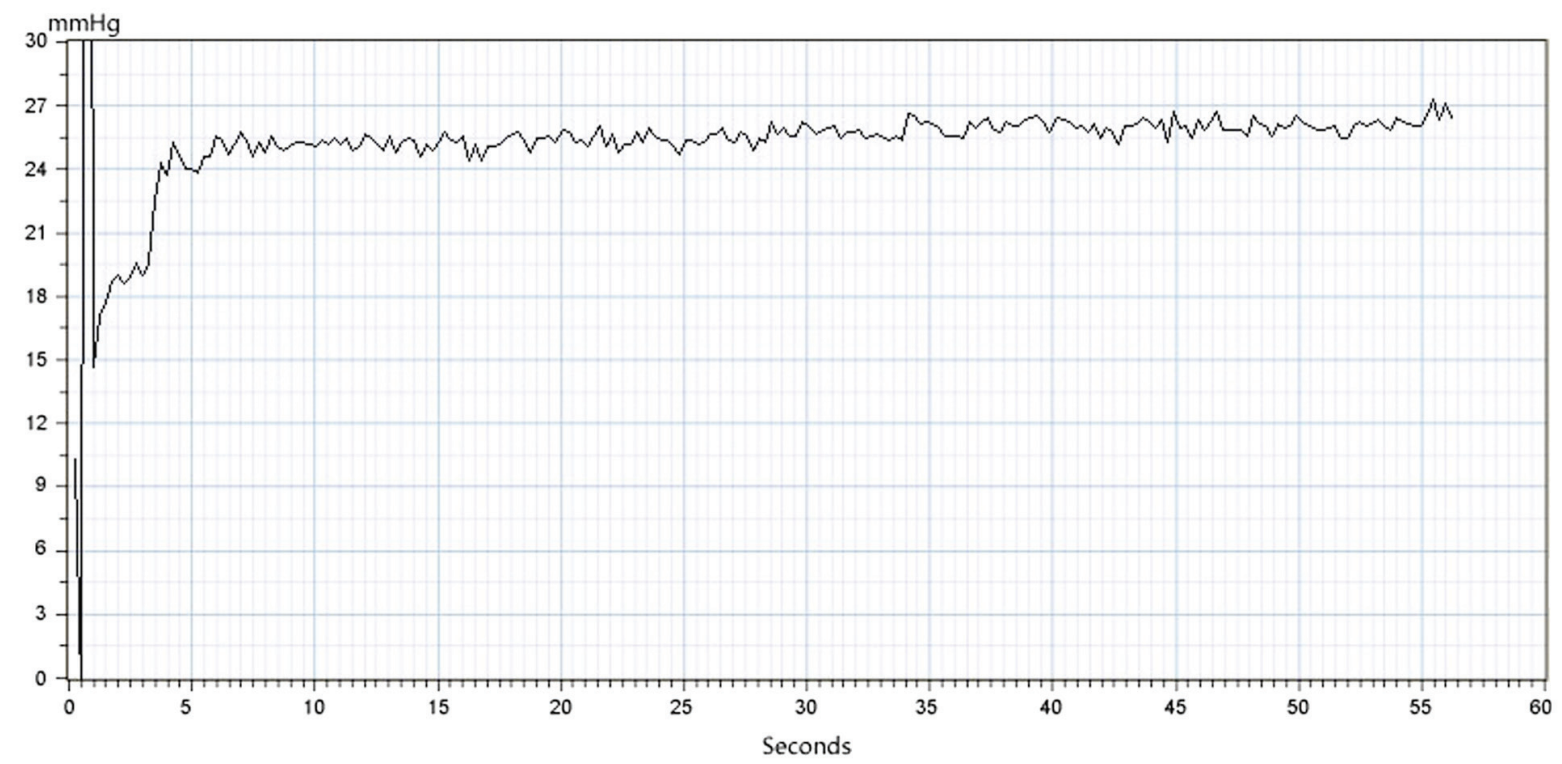

Figure 3. Endoscopic variceal pressure recording.

\section{Results}

The esophageal variceal pressure results of the patients were obtained successfully and there were no intraoperative or postoperative complications, including bleeding. Although the accuracy of results was affected by esophageal peristalsis or additional esophageal peristalsis created by breathing and coughing, this effect could be minimized by sedation, antispasmodic and sizing the intermission between two peristaltic waves in future studies.

The mean variceal pressure value of the 80 patients included in the present study was $14.2 \pm 2.2 \mathrm{mmHg}$, and the mean PPG value was $28.0 \pm 4.7 \mathrm{mmHg}$. There was a linear correlation between the variceal pressure and PPG values ( $r=0.940, P<0.001$, Fig. 4). The PPG values were higher than the variceal pressure values $(\mathrm{t}=23.86, \mathrm{P}<0.001)$. In addition, there was an association between the PPG and variceal pressure in open TIPS conditions $(20.7 \pm 4.8$ and $10.5 \pm 2.3 \mathrm{mmHg}$, respectively, $\mathrm{r}=0.979, \mathrm{P}<0.001$, Fig. 5). The
PPG values were higher than the variceal pressure values $(\mathrm{t}=17.15, \mathrm{P}<0.001)$. Intra- and inter-observer agreement was determined by the correlation coefficient. The intra-observer correlation for variceal pressure measurements was 0.94 , and the inter-observer correlation for variceal pressure measurements was 0.96 .

\section{Discussion}

It is well-established that variceal pressure exerts a pivotal function in predicting variceal bleeding and is imperative when assessing the effect of portal hypertension pharmacotherapy. There are various techniques to measure variceal pressure; one is by directly puncturing the varix with a thin needle, whereas other methods involve using non-invasive techniques. The application of the former is restricted due to the high risk of variceal bleeding, therefore, the present study investigated a fiber-optic pressure sensor as a novel non-invasive technique to measure variceal pressure. The results of the present study 
Table I. Demographic and clinic profile of the study population.

\begin{tabular}{lc}
\hline Characteristic & $\mathrm{n}(\%)$ \\
\hline Total patients & 80 \\
Age (years) & $50.9 \pm 10.9$ \\
Sex & \\
Male & $61(76.2)$ \\
Female & $19(23.8)$ \\
Etiology & \\
Viral & $64(80)$ \\
Alcohol & $2(2.5)$ \\
Primary biliary cirrhosis & $3(3.8)$ \\
Other & $11(13.7)$ \\
Child-Pugh score & \\
A & $15(18.8)$ \\
B & $46(57.4)$ \\
C & $19(23.8)$ \\
Varix grade & \\
F2 & $22(27.5)$ \\
F3 & $58(72.5)$ \\
Variceal pressure (mm Hg) & $14.2 \pm 2.2$ \\
PPG (mm Hg) & $28.0 \pm 4.7$ \\
\hline
\end{tabular}

PPG, portal pressure gradient.

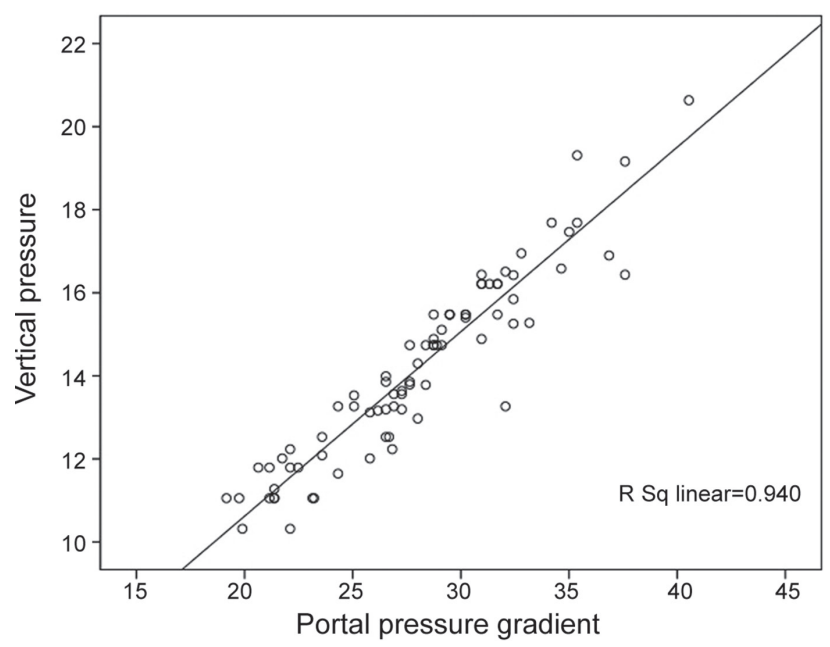

Figure 4. Correlation between variceal pressure and PPG prior to the transjugular intrahepatic stent-shunt procedure. PPG, portal pressure gradient.

indicated that this technique can predict the risk of bleeding in patients with liver cirrhosis and may also be used to monitor drug efficacy. Screening for high-risk patients with cirrhosis of the liver would assist in providing individualized treatment strategies for patients with cirrhosis and in developing a standardized treatment. Therefore, the present study evaluated the accuracy and reproducibility of a fiber-optic pressure sensor, to measure its suitability in a clinical setting.

The level of portal hypertension is an important indicator of the development of variceal bleeding, and portal hypertension

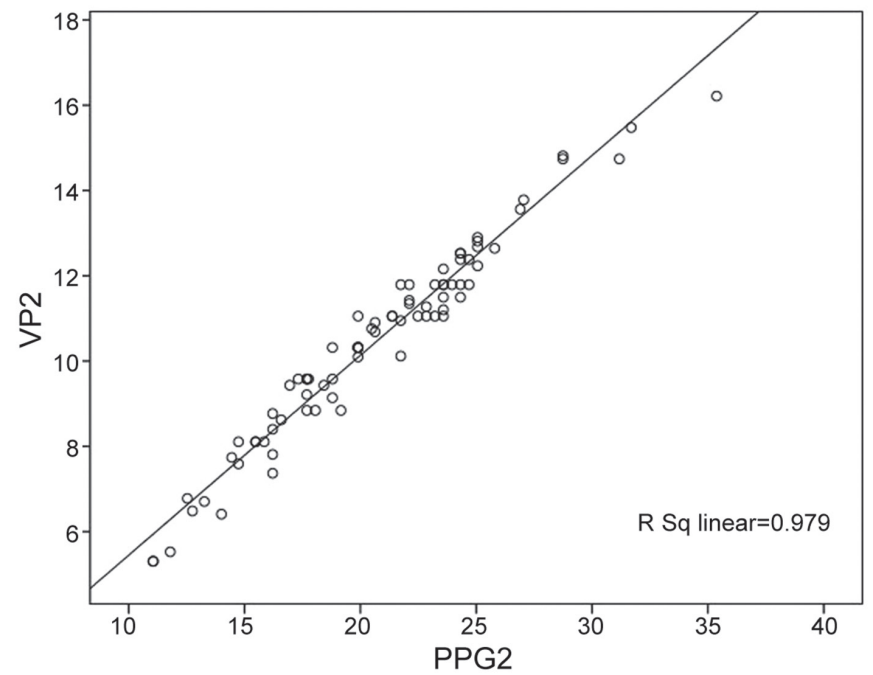

Figure 5. Correlation between VP and PPG with the open transjugular intrahepatic stent-shunt procedure. PPG, portal pressure gradient; VP, variceal pressure.

is generally evaluated by determining the hepatic venous pressure gradient (HVPG) (12). However, measuring the HVPG is invasive and inconvenient, which has limited its applications as a routine method. Approaches that aim to assess variceal pressure using non-invasive techniques may provide a reasonable alternative method to HPVG. As HVPG is closely associated with variceal pressure, measuring the variceal pressure should provide an accurate evaluation of the HVPG. Previous studies have demonstrated that the measurement of variceal pressure using non-invasive techniques was positively correlated with those measured by direct puncture and HVPG $(13,14)$. In order to examine the reliability of the device based on a fiber-optic pressure sensor, the present study used the PPG, which was measured directly by the catheterization of portal veins undergoing TIPS in patients with cirrhosis as a reference control. A correlation was observed between the variceal pressure measured using the device and PPG, and a good positive correlation was identified between them. Therefore, measuring variceal pressure using the device enabled evaluation of the degree of portal hypertension in a non-invasive and convenient manner.

The measurement of variceal pressure in the present study was technically satisfactory in 78 patients. The high rate of success in measurement was due to the small measuring surface of the fiber-optic pressure sensor ensuring its suitability for measuring variceal pressure in varices of small size. Good and steady contact between the fiber-optic pressure sensor and the varices enabled a stable tracing of variceal pressure to be obtained

The results of the present study demonstrated that the measurement of variceal pressure based on a fiber-optic pressure sensor required only short-term training under gastrointestinal endoscopy. As with a biopsy, the fiber-optic pressure sensor can be easily applied over any esophageal varix through the endoscopic working channel described in our previous study (10). The pressure tracing on the device in vivo was characterized by a sharp upstroke and stable plateau phase. 
In the present study, it was observed that variceal pressure was significantly correlated with portal pressure, and intra-observer and inter-observer agreement in the measurement of variceal pressure was good. As hypothesized, the present study also demonstrated that variceal pressure was significantly lower than PPG, potentially as a result of the significant resistance in venous circulation in collateral vessels. It has been stated previously that $6 \mathrm{mmHg}$ in esophageal varices is ${ }^{3} 12 \mathrm{mmHg}$ in HVPG, owning to the extra pressure scatters in palisade esophageal varices (8).

To the best of our knowledge, the device constructed herein has the smallest gauge for measuring variceal pressure, which can pass through the working channel of a standard endoscope. In addition, the small measuring surface makes the device suitable for measuring variceal pressure in small-radius vessels. However, in the present study, the measurement of variceal pressure was not satisfactory in two patients due to poor contact between the device and the varix. In a previous study, Bosch et al (15) first introduced a pressure gauge fixed to the tip of an endoscope for measuring variceal pressure. The pressure gauge consisted of a small chamber covered by a thin latex membrane, which was perfused with a constant flow of nitrogen. According to Newtonian force balance equations, it is expected that when the gauge is applied over the varix, the pressure required to perfuse the gauge measured by a sensitive pressure transducer is equivalent to the pressure inside the varix. Using an in vitro experiment, the accuracy and reproducibility of the Varipress were considered satisfactory. However, limitations of this technique have been reported in varices of a small size. In addition, the reliability of the Varipress in vivo remains controversial, as ideal placement of the gauge on the varix may be difficult and patient-dependent factors may influence variceal pressure measurements. It was estimated that $\sim 25 \%$ of patients initially scheduled for variceal pressure measurements must be excluded due to poor contact between the gauge and the varices that result in unstable pressure values, particularly for varices of a small size $(4,16,17)$.

As the fiber-optic pressure sensor device often suffers from thermal stability issues, the variceal pressures measured by the device are susceptible to influence from circumstantial temperature. Prior to measuring variceal pressure, the zero reference of the device must be set in order to acclimatize to the temperature at the time. Another disadvantage of this method is that in the dynamic process of observing the varicose veins being indented, the human eye determines that the varicose veins have been depressed; therefore, the pressure measurement technique is dependent upon the observations of the researcher administering the technique.

Despite the aforementioned limitations, the present study provides support for the use of this novel device based on a fiber-optic pressure sensor, owing to the fiber-optic pressure sensor being more convenient than other non-invasive techniques and having a short training period. Its accuracy is higher for small size varices than those of other sizes. However, in order to evaluate clinical applicability of the novel device, further investigation is required in a larger study cohort. Taken together, the results of the present study demonstrated that the device and method investigated herein was accurate and precise for evaluating the risk of variceal bleeding and efficacy of drug therapy, and was reliable when detecting variceal pressure.

\section{Acknowledgements}

Not applicable.

\section{Funding}

The present study was supported by a grant from the General Program of National Natural Science Foundation of China (grant no. 81271736). The funders had no role in study design, data collection and analysis, decision to publish or preparation of the manuscript.

\section{Availability of data and materials}

The datasets used and/or analyzed during the present study are available from the corresponding author on reasonable request.

\section{Authors' contributions}

DK provided technical support and designed the study. XW performed the experiments, collected and analyzed the data and contributed substantially to revision of the manuscript. HL and JW performed the experiments, collected and analyzed the data and wrote the manuscript. AW provided detailed instructions on the principle of the method and was involved in modification of the methods. All authors hxave read and approved the final manuscript.

\section{Ethics approval and consent to participate}

The study protocol was authorized by the Ethics Committee of Anhui Medical University (Hefei, China) and all patients provided written informed consent prior to study inclusion. The trial was registered with the Chinese Clinical Trial Registry (A new device of fiber-optic pressure sensor for measuring variceal pressure-ChiCTR1800016926 17/06/2018).

\section{Patient consent for publication}

Not applicable.

\section{Competing interests}

The authors declare that they have no competing interests.

\section{References}

1. Scaglione S, Kliethermes S, Cao G, Shoham D, Durazo R, Luke A and Volk ML: The epidemiology of cirrhosis in the United States: A population-based study. J Clin Gastroenterol 49: 690-696, 2015.

2. Tandon RK and Saikia N: Measuring intravariceal pressure. Gastrointest Endosc 70: 414-416, 2009.

3. Palmer ED: On correlations between portal venous pressure and the size and extent of esophageal varices in portal cirrhosis. Ann Surg 138: 741-744, 1953.

4. Rigau J, Bosch J, Bordas JM, Navasa M, Mastai R, Kravetz D, Bruix J, Feu F and Rodés J: Endoscopic measurement of variceal pressure in cirrhosis: Correlation with portal pressure and variceal hemorrhage. Gastroenterology 96: 873-880, 1989.

5. Gertsch PH and Meister JJ: Pressure measurement in oesophageal varices: Preliminary report on a new non-invasive method. Gut 28: 1162-1165, 1987. 
6. Miller ES, Kim JK, Gandehok J, Hara M, Dai Q, Malik A, Miller A and Miller L: A new device for measuring esophageal variceal pressure. Gastrointest Endosc 56: 284-291, 2002.

7. Miller LS, Dai Q, Thomas A, Chung CY, Park J, Irizarry S, Nguyen T, Thangada V, Miller ES and Kim JK: A new ultrasound-guided esophageal variceal pressure-measuring device. Am J Gastroenterol 99: 1267-1273, 2004.

8. Vegesna AK, Chung CY, Bajaj A, Tiwana MI, Rishikesh R, Hamid I, Kalra A, Korimilli A, Patel S, Mamoon R, et al: Minimally invasive measurement of esophageal variceal pressure and wall tension (with video). Gastrointest Endosc 70: 407-413, 2009.

9. Kong DR, Xu JM, Zhang L, Zhang C, Fu ZQ, He BB, Sun B and Xie Y: Computerized endoscopic balloon manometry to detect esophageal variceal pressure. Endoscopy 41: 415-420, 2009.

10. Kong DR, He BB, Wu AJ, Wang JG, Yu FF and Xu JM: Fiberoptic sensor for noninvasive measurement of variceal pressure. Endoscopy 45 Suppl 2 UCTN: E55-E56, 2013.

11. Tajiri T, Yoshida H, Obara K, Onji M, Kage M, Kitano S, Kokudo N, Kokubu S, Sakaida I, Sata M, et al: General rules for recording endoscopic findings of esophagogastric varices (2nd edition). Dig Endosc 22: 1-9, 2010.

12. Garcia-Tsao G, Abraldes JG, Berzigotti A and Bosch J: Portal hypertensive bleeding in cirrhosis: Risk stratification, diagnosis, and management: 2016 practice guidance by the American Association for the study of liver diseases. Hepatology 65: 310-335, 2017.
13. Watari A, Miyata K, Kanazawa H and Kobayashi M: Comparison of portal pressure with intravascular esophageal variceal pressure (IEVP) directly measured with a flexible indwelling needle. Gastroenterol Jpn 28: 631-637, 1993.

14. Gertsch P, Fischer G, Kleber G, Wheatley AM, Geigenberger G and Sauerbruch T: Manometry of esophageal varices: Comparison of an endoscopic balloon technique with needle puncture. Gastroenterology 105: 1159-1166, 1993.

15. Bosch J, Bordas JM, Rigan J, Viola C, Mastai R, Kravetz D, Navasa M and Rodés J: Noninvasive measurement of the pressure of esophageal varices using an endoscopic gauge: Comparison with measurements by variceal puncture in patients undergoing endoscopic sclerotherapy. Gastroenterology 6: 667-672, 1986.

16. Polio J, Hanson J, Sikuler E, Vogel G, Gusberg R, Fisher R and Groszmann RJ: Critical evaluation of a pressure-sensitive capsule for measurement of esophageal varix pressure. Studies in vitro and in canine mesenteric vessels. Gastroenterology 92: 1109-1115, 1987.

17. Puckett JL, Liu J, Bhalla V, Kravetz D, Krinsky ML, Hassanein T and Mittal RK: Ultrasound system to measure esophageal varix pressure: An in vitro validation study. Am J Physiol Gastrointest Liver Physiol 288: G914-G919, 2005.

This work is licensed under a Creative Commons Attribution-NonCommercial-NoDerivatives 4.0 International (CC BY-NC-ND 4.0) License. 